\title{
Preventing female genital mutilation in high income countries: a systematic review of the evidence
}

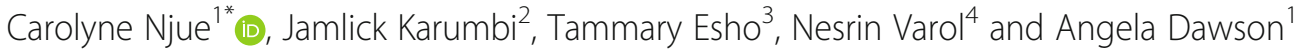

\begin{abstract}
Background: Female genital mutilation (FGM) is prevalent in communities of migration. Given the harmful effects of the practice and its illegal status in many countries, there have been concerted primary, secondary and tertiary prevention efforts to protect girls from FGM. However, there is paucity of evidence concerning useful strategies and approaches to prevent FGM and improve the health and social outcomes of affected women and girls.
\end{abstract}

Methods: We analysed peer-reviewed and grey literature to extract the evidence for FGM prevention interventions from a public health perspective in high income countries by a systematic search of bibliographic databases and websites using appropriate keywords. Identified publications were screened against selection criteria, following the PRISMA guidelines. We examined the characteristics of prevention interventions, including their programmatic approaches and strategies, target audiences and evaluation findings using an apriori template.

Findings: Eleven documents included in this review described primary and secondary prevention activities. High income countries have given attention to legislative action, bureaucratic interventions to address social injustice and protect those at risk of FGM, alongside prevention activities that favour health persuasion, foster engagement with the local community through outreach and the involvement of community champions, healthcare professional training and capacity strengthening. Study types are largely process evaluations that include measures of short-term outcomes (pre- and post-changes in attitude, knowledge and confidence or audits of practices). There is a dearth of evaluative research focused on empowerment-oriented preventative activities that involve individual women and girls who are affected by FGM. Beattie's framework provides a useful way of articulating negotiated and authoritative prevention actions required to address FGM at national and local levels.

Conclusion: FGM is a complex and deeply rooted sociocultural issue that requires a multifaceted response that encompasses socio-economic, physical and environmental factors, education and learning, health services and facilities, and community mobilisation activities. Investment in the rigorous longitudinal evaluation of FGM health prevention efforts are needed to provide strong evidence of impact to guide future decision making. A national evidence-based framework would bring logic, clarity, comprehension, evidence and economically more effective response for current and future prevention interventions addressing FGM in high income countries.

Keywords: Female genital mutilation, Migrants, Health prevention, Systematic review, High-income countries

\footnotetext{
* Correspondence: Carolyne.Njue@uts.edu.au

${ }^{1}$ The Australian Centre for Public and Population Health Research, Faculty of

Public Health, University of Technology Sydney, PO Box 123, Broadway,

Sydney, NSW 2007, Australia

Full list of author information is available at the end of the article
}

(C) The Author(s). 2019 Open Access This article is distributed under the terms of the Creative Commons Attribution 4.0 International License (http://creativecommons.org/licenses/by/4.0/), which permits unrestricted use, distribution, and reproduction in any medium, provided you give appropriate credit to the original author(s) and the source, provide a link to the Creative Commons license, and indicate if changes were made. The Creative Commons Public Domain Dedication waiver (http://creativecommons.org/publicdomain/zero/1.0/) applies to the data made available in this article, unless otherwise stated. 


\section{Plain English summary}

Female Genital Mutilation (FGM) is a cultural practice that is associated with poor health outcomes. The migration of women and girls with FGM to countries such as the United Kingdom, the United States of America and Australia, where FGM is not traditionally practiced, has led to the development of programs to prevent FGM and care for affected women and girls. The purpose of this study is to identify and review existing literature to find out what strategies and approaches are useful to prevent FGM and improve the health of affected women and girls. The majority of the documents we included in this review described laws developed to prevent FGM and safeguard girls and women, as well as media campaigns and health education activities for health professionals and community members. All the evaluations measured short term outcomes such as improvements in knowledge and we did not find any evidence of long term changes. There is a need to carry out well designed evaluations to understand how interventions can change behaviour. This evidence can then be used to inform national plans to prevent FGM.

\section{Background}

WHO defines female genital mutilation (FGM) as "all procedures that involve the partial or total removal of the external female genitalia, or other injury to the female genital organs for nonmedical reasons" [1]. WHO classifies FGM into four categories with type III (infibulation) being the most severe form [1]. This is a more detailed description of practices than the one offered by UNICEF [2]. FGM, also known as female genital cutting or female circumcision, has serious health consequences that may lead to death from haemorrhage and/or infection, urinary and genital tract infections, gynaecological, obstetric, sexual and psycho-social complications or death from haemorrhage [3-5]. While the prevalence of FGM is decreasing and varies across countries [6] UNICEF has estimated that more than 200 million of girls and women have undergone Female Genital Mutilation (FGM) globally and three million girls may be at risk of undergoing FGM every year [7]. FGM occurs in more than 40 countries throughout the world. It is practiced by communities in 28 African countries, communities in the southern parts of the Arabian Peninsula and along the Persian Gulf and in communities in India, Indonesia and Malaysia [8, 9].

Difficult economic conditions and conflict are among the factors that have resulted in increasing migration of people from FGM prevalent nations to high income countries (HIC) where FGM is not traditionally practiced such as the United States of America (USA), United Kingdom, Australia, New Zealand, and across Europe [9]. Many of these women and girls would be at risk of undergoing FGM in their countries of origin. Some migrants have continued the practice in their countries of migration, with the aim of maintaining their culture and identity $[10,11]$. However, there is evidence that migration to countries where FGM is not prevalent has a positive influence on the abandonment of this practice [12-16]. Some of the reasons cited for this change include improved knowledge of the health consequences and weakening of social pressure to undertake FGM that is associated with changing perceptions regarding the benefits of the practice for marriageability, religious observance and social acceptance. Moreover, those affected by FGM may be stigmatised in their new country of migration [17]. FGM is a prosecutable offence in most HIC. Whilst this may dissuade communities from undertaking FGM, it may also encourage families to conduct FGM in secret or take their children overseas to be circumcised.

The exact number of women and girls living with FGM in HIC is largely unknown due to the sensitive nature of the issue and the lack of routinely collected data. However, it is estimated that almost half a million women living in Europe have been subjected to the practice [18]. In the United Kingdom (UK) and USA, based on 2015 estimates, there are about 137,000 women and girls who have undergone FGM and 507,000 who are at risk of FGM [19, 20]. Health professionals in HIC are often not aware of FGM or have knowledge and skills to adequately care for affected women or protect children at risk. FGM therefore presents a challenge to the health system in HIC [21, 22].

Given the harmful effects of this practice and its illegal status in many countries, there have been concerted efforts to prevent FGM in HIC through advocacy and other prevention activities including education, information and public communication campaigns for affected women and girls. Mandatory reporting protocols have been developed in some countries, such as the United Kingdom (UK), to protect women and girls at risk. In addition, there are police protocols and legal means to prosecute those suspected of carrying out the practice [23] However, there is not enough evidence concerning effective strategies and approaches to prevent FGM and improve the health and social outcomes for affected women and girls. Four recent reviews have largely focused on interventions from African countries [24-27]. We therefore undertook a systematic review to identify the evidence for FGM prevention interventions from a public health perspective in HIC. Specifically, we examined the characteristics of interventions, including their programmatic approaches and strategies, target audiences and evaluation findings. The review aims to contribute to improving the knowledge base to inform the design and evaluation of FGM health interventions to 
prevent the practice and optimize health outcomes for girls and women with FGM.

\section{Methods}

Due to the short-term nature of the largely process evaluations we identified that used a range of methodologies, we were not able to pool data to establish the overall effect of the interventions on prevalence. This determined the use of a qualitative content analysis rather than a quantitative meta-analysis to establish effectiveness. We undertook a systematic review and content analysis using an apriori framework to analyse the characteristics and underpinning approaches of interventions to prevent FGM in HIC and their outcomes. This review was registered with the International Prospective Register of Systematic Reviews as PROSPERO CRD42018092299.

For the purposes of this review, the term HIC is based on the World Bank definition, which divides member countries into different income groups. A HIC is as a country with a higher gross national income per capita of $\$ 12,376$ USD [28]. We defined an 'FGM intervention' as any form of action or deliberate process to interfere with, modify or change individuals' or groups of people's knowledge, attitudes or behaviours to prevent FGM and thereby reduce the prevalence and improve the health outcomes of girls and women affected by FGM. We used Tannahill's health promotion approach [29] to define types of interventions for inclusion and as such, clinical interventions were excluded. However, education programs to support affected women who may have undergone de-infibulation for example, were part of this review as was legislation to protect girls and community awareness to prevent FGM. We selected Tannahill's model of health promotion where prevention is a key pillar [29] because it acknowledges, as other authors have noted, that prevention cannot be achieved without health promotion [30]. We aimed to understand the different strategies that are used to prevent FGM that reveal power dynamics and possible ethical dilemmas. This understanding informed the use of Bettie's model [31] to further explore these strategies that recognises that prevention and health promotion are conceptually linked.

To achieve a comprehensive systematic search and hence retrieve all contemporary empirical evidence, we assessed all types of literature, ranging from grey literature reports to peer-reviewed literature concerned with the evaluation of interventions aimed at preventing and/ or providing education for girls and women with FGM in HIC. The search for evidence was limited to what was available online and snowballing from bibliographies of retrieved articles.

A set of keywords used in the search were 'female genital mutilation/cutting, 'female genital mutilation', 'female genital cutting,' 'female circumcision,' 'clitoridectomy,' 'excision,' 'infibulation,' 'sunna,' 'FGM intervention,' 'FGM/FGC, 'FGM or FGC program, 'high income country,' developed country', and 'developed regions'. Peer reviewed literature was identified using PubMed and CINHAL (EBSCO), EMBASE and Web of Science. We further searched institutional websites and databases of organizations involved in FGM activities, to identify reports and any possible "grey literature." We conducted general searches using Google and Google Scholar to ensure no literature was missed. In addition, we cross-referenced articles, which were relevant and scanned through their bibliography to identify additional materials.

The first author $(\mathrm{CN})$ identified studies for relevance based on the title and abstract and the third author (TE) repeated the process to ensure no relevant studies were excluded. We included studies that focus on assessments or evaluations of FGM interventions published since 2000 as being eligible. We used a pre-established eligibility and inclusion and exclusion criteria to guide the screening and selection process. The inclusion criteria used were the following: (i) countries described were from a HIC setting as defined above; (ii) were conducted in recent years (January 2000-March 2018); (iii) the studies focused on assessing the impact of FGM interventions; and (iv) were in English. There was no restriction; all study types, designs and methodologies, those appearing in peer review journal or in grey literature from a recognised institution and/or government, or a $\mathrm{PhD}$ thesis, were all included so long as there was a clear methodology to enable an assessment of quality. Studies were excluded if they were from low or middle-income countries (LMIC), if they assessed consequences of FGM, described national action plans, were comprised of theoretical notes and recommendations/guidelines, or if they were not in English. Our search located 87 study reports that were eligible for review as shown in the PRISMA flow chart [32] (Fig. 1).

Following the screening, 42 study reports were identified as eligible for full text review. These were retrieved and assessed by the second author (JK) and counterchecked by first author $(\mathrm{CN})$. The authors $(\mathrm{JK}$ and $\mathrm{CN}$ ) independently appraised the studies for quality of reporting, this included an assessment of its quality, size and consistency of the body of evidence (See Tables 1 and 2). The study type, design, and methodology determined the type of quality assessment tool used and data were recorded on Microsoft Excel software.

Of the 42 documents, 11 met the inclusion criteria (See Table 3). Evidence from ten grey literature was assessed for quality using Authority, Accuracy, Coverage, Objectivity, Date, and Significance (AACODS) Checklist [45]. We used Critical Appraisal Skills Programme (CASP) checklists to appraise one study published in peer-reviewed journals [46]. Data was extracted from the findings' sections of the included papers (ad-verbatim 


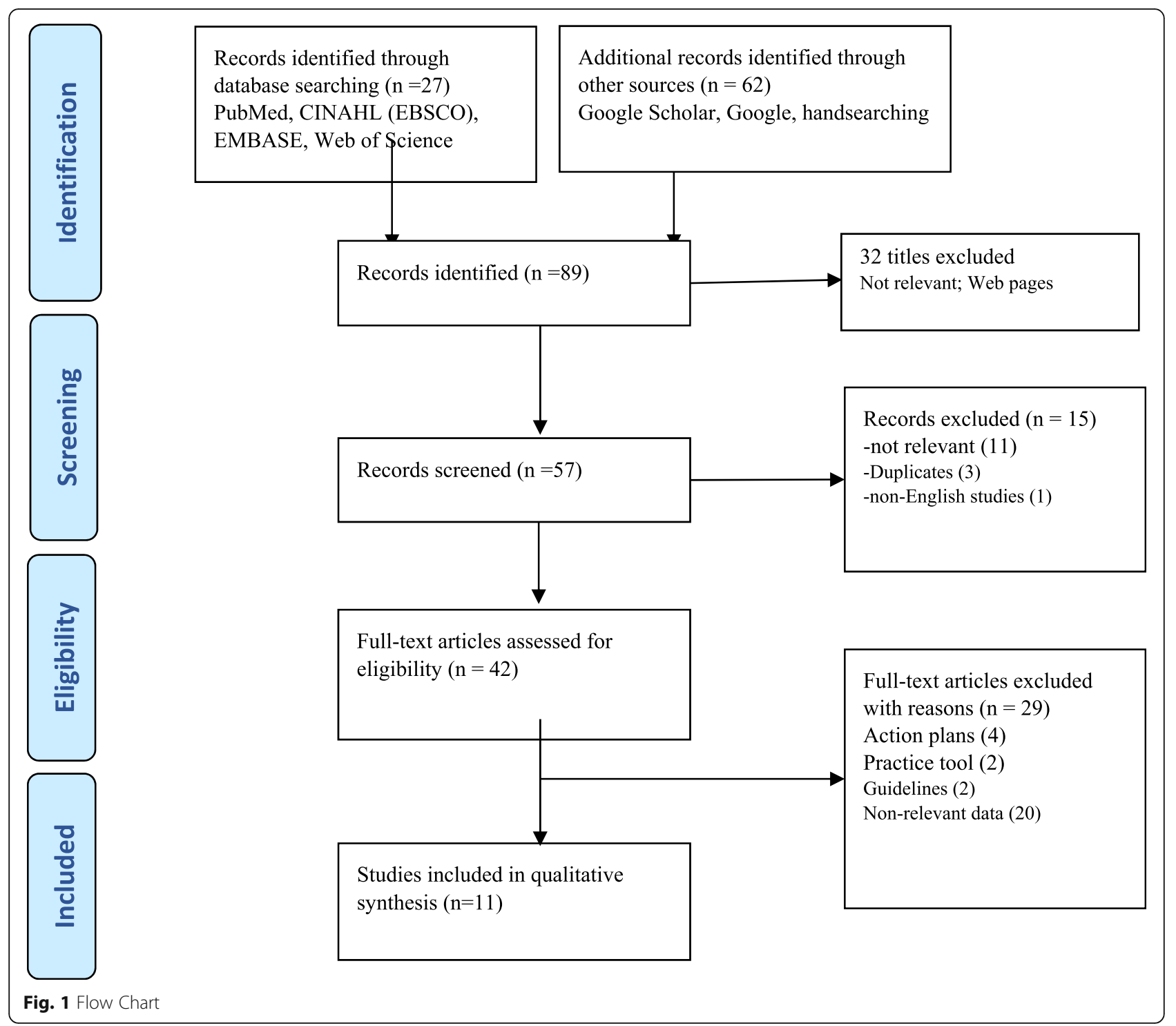

and marked in quotation marks) in the form of sentences, phrases, or text units dealing the effectiveness of an intervention to identify patterns according to each intervention. Data extraction was undertaken by the authors $(\mathrm{CN}$ and $\mathrm{JK})$ using a predesigned table. This was counterchecked by fourth (NV) and fifth (AD) authors.

We first categorized the FGM interventions studies according to different levels of prevention, namely: primary, secondary and tertiary, as per the public health approach [47] and examined the outcomes of each initiative. In addition to the levels of prevention and the associated outcomes, we then undertook a content analysis informed by Beattie's model of health promotion to understand dimensions of power and the socio-cultural context of the interventions. Drawing on this model, we examined the mode and focus of interventions specifically how negotiated action was achieved or how authoritative interventions were successful at changing individual or group behaviour [31]. We mapped this across the four quadrants of the model.

\section{Results}

A total of 11 study reports are included in this review produced across multiple agencies involved in delivering FGM programs in HIC. One study [33] discusses FGM programs across 19 HIC. Five reports discuss FGM programs in the UK $[34,35,39,40,44]$. Three reports outline work undertaken in EU member states [37, 38, 48] and two documents describe FGM efforts in Canada [42] \{Daniel, 2014 \#229\} and Australia [41] \{Scott, 2011 $\# 220\}$. The 11 studies used qualitative and quantitative methods. Six studies were judged to be of high quality (five of these were based on the AACODs checklist) [37-39, 42, 48]. Five studies were judged to be of 


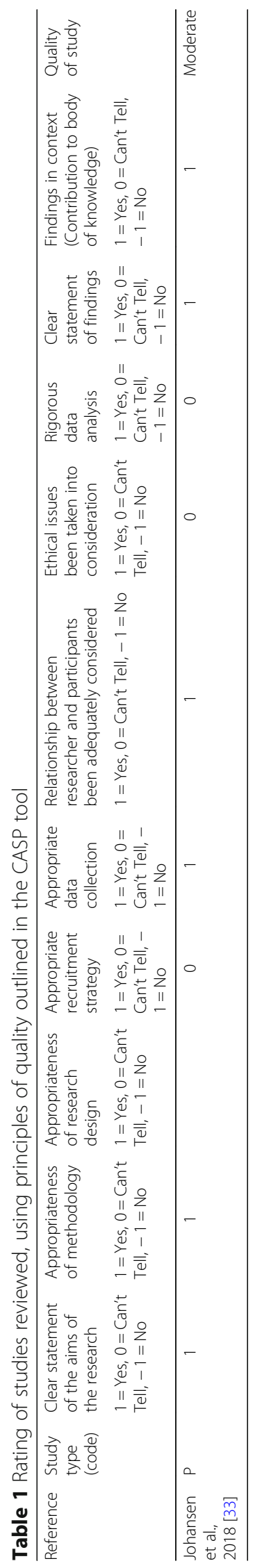




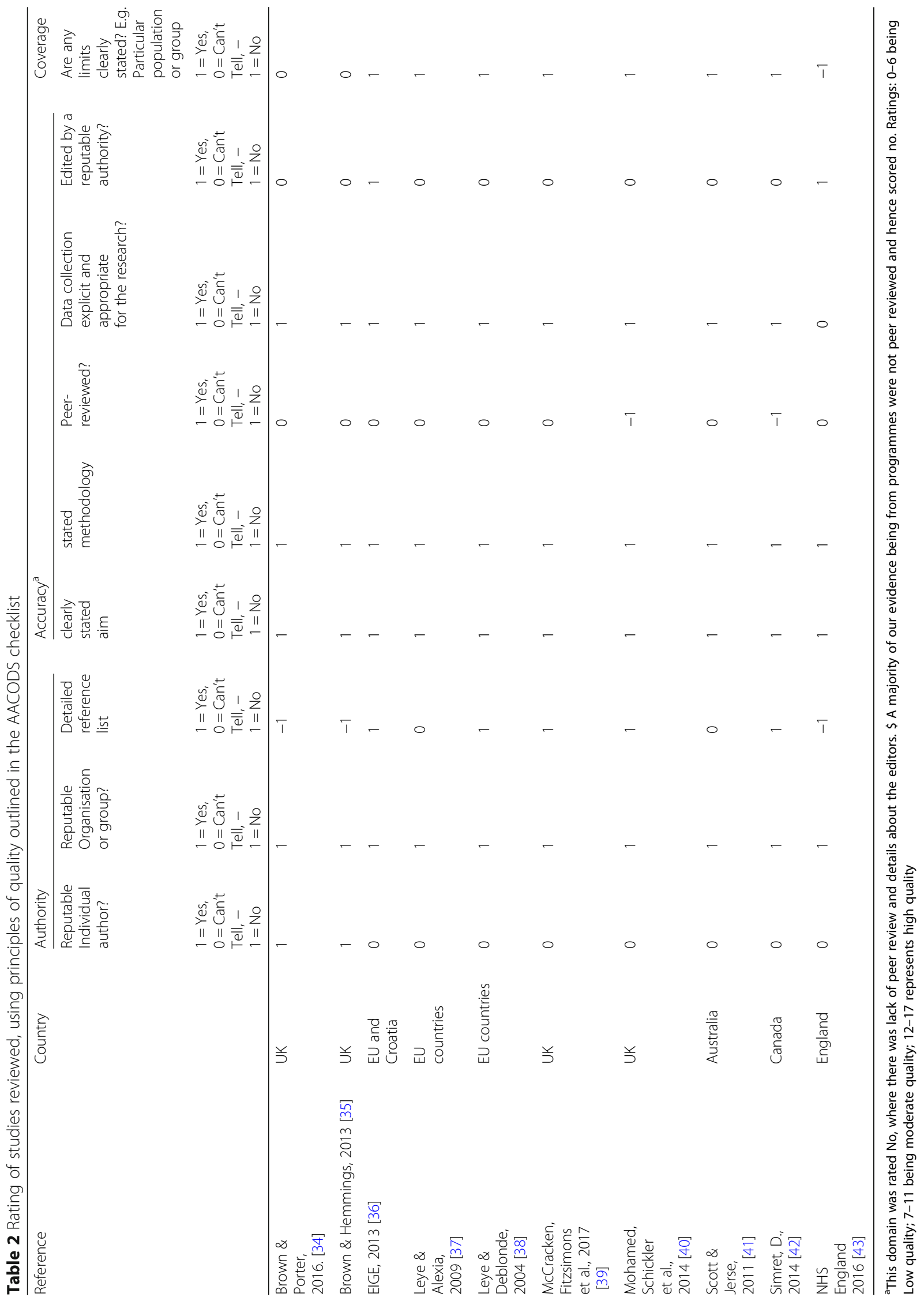




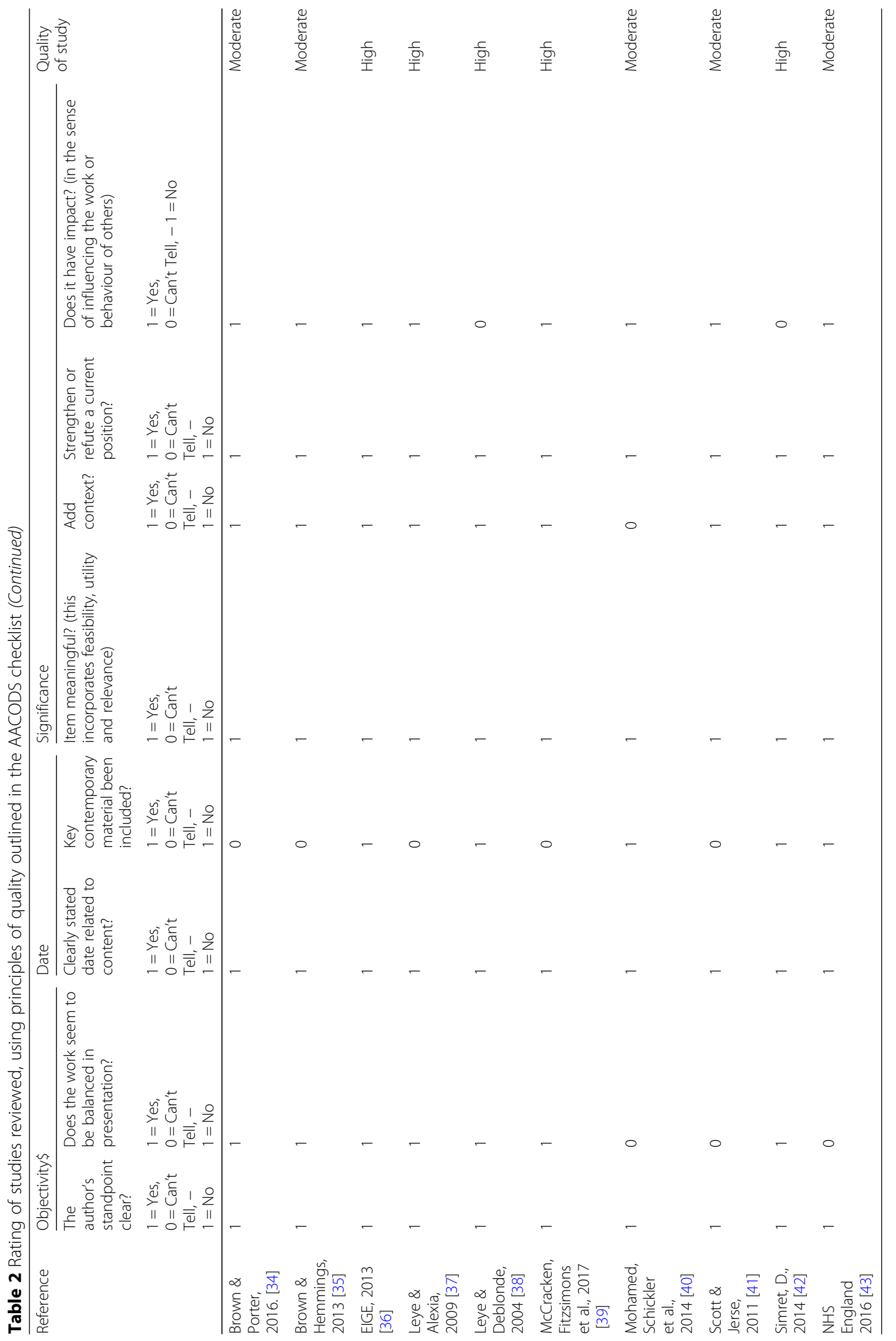




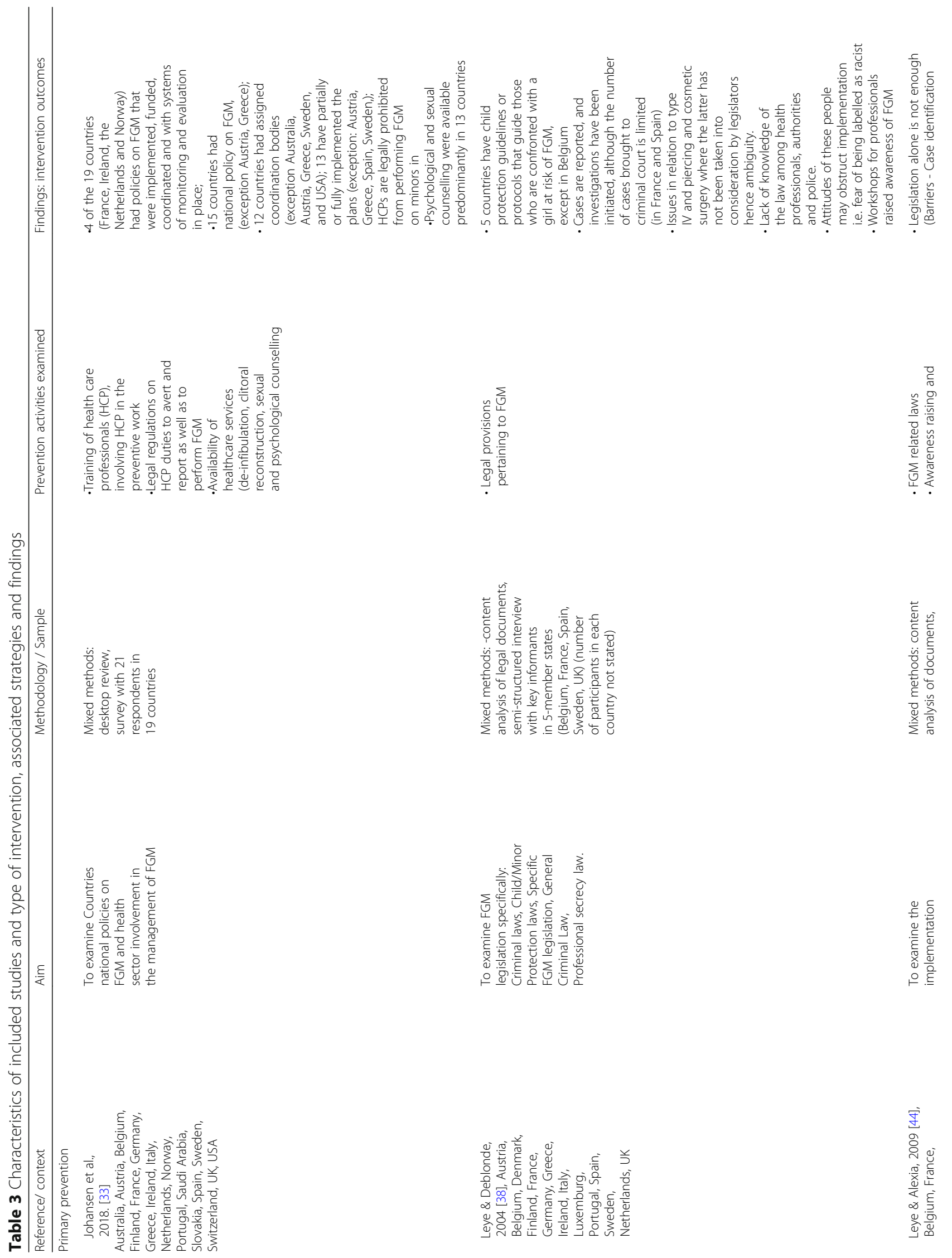




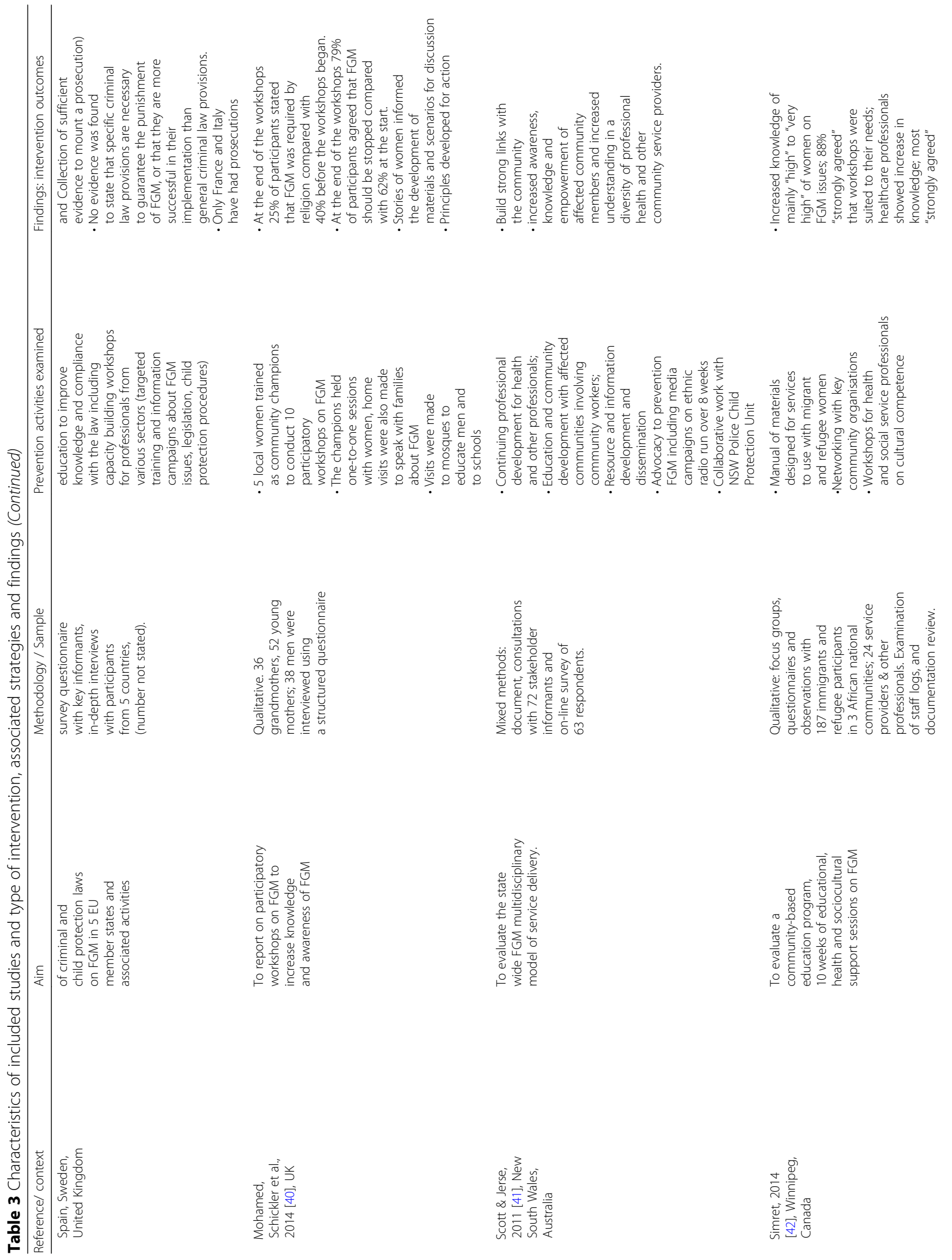



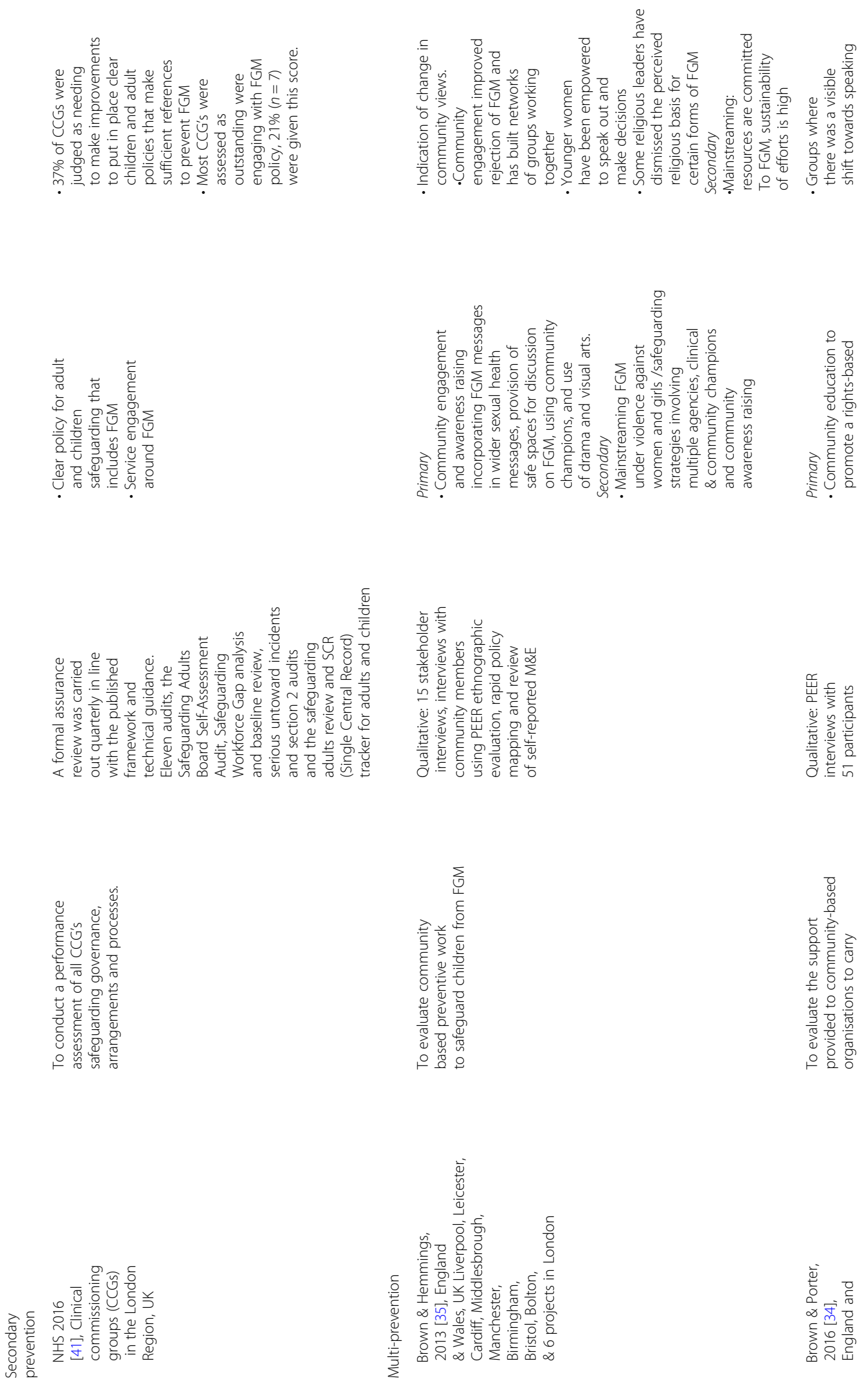

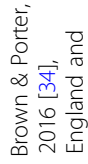




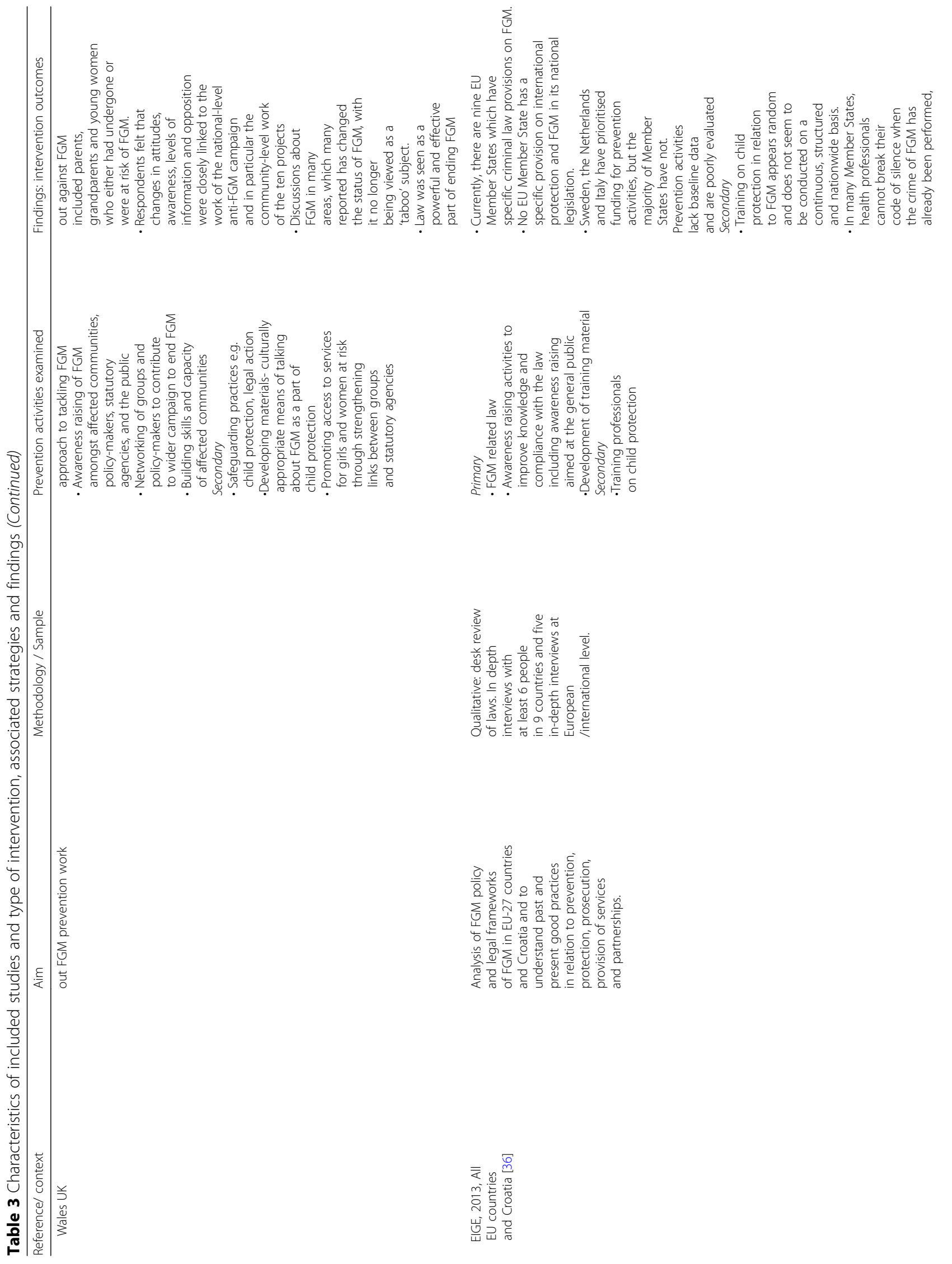




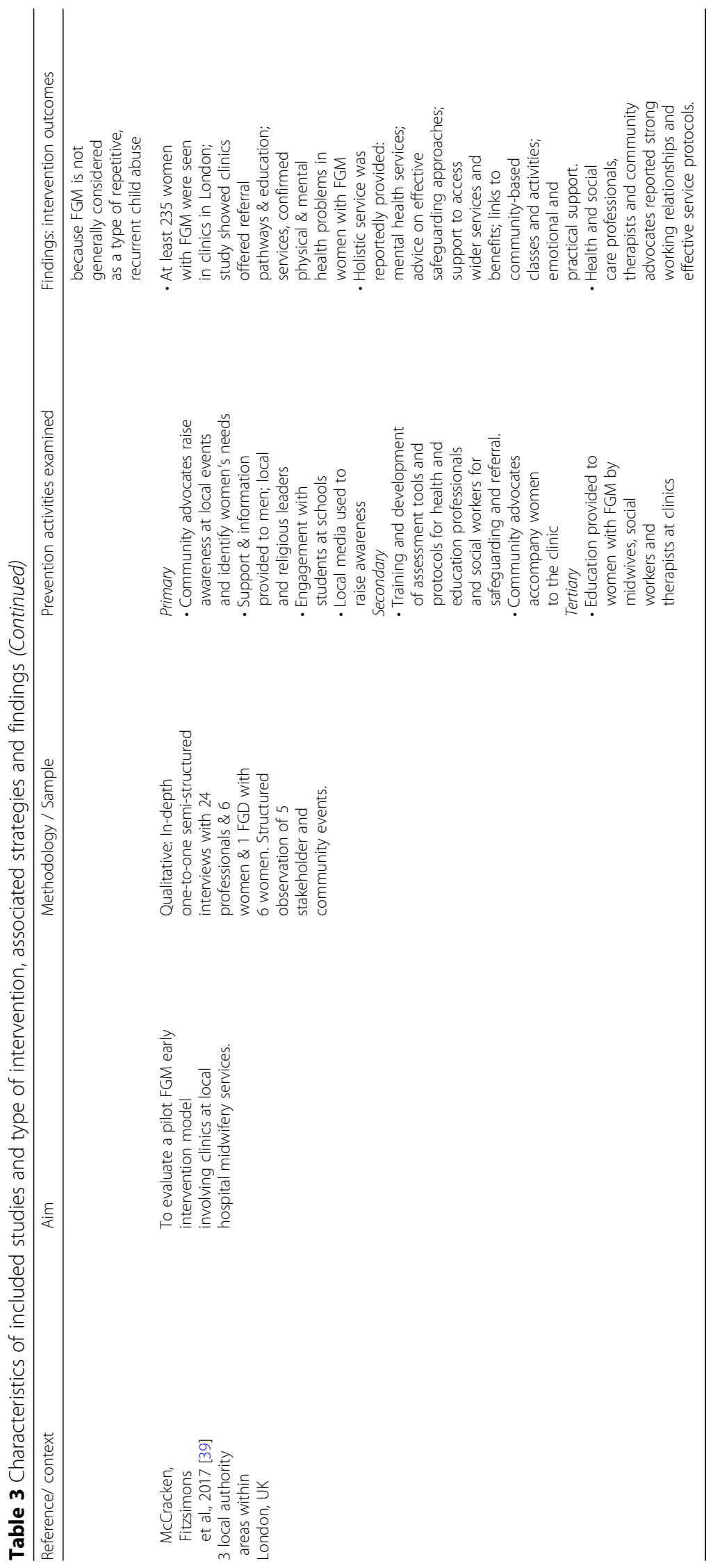


moderate quality (three of these were based on the AACODs checklist) $[35,40,41]$ and one was based on the CASP checklist for qualitative studies [33] \{Abreu, $2015 \# 222\}$. One study was judged to be of low quality based on the AACODs checklist [44]. The activities described in the 11 reports were classified according to their primary, secondary or tertiary prevention focus (Summary Table 2).

\section{Interventions focused on the primary prevention of FGM}

All documents included in the review described primary prevention activities that sought to increase individual professional and community awareness and understanding of FGM as an infringement on human rights, as well as the adverse health outcomes associated with the practice and the law as it pertained to FGM. Studies also provided insight into the current legal status of FGM in a number of European countries and issues relating to the implementation of the law.

Mohammed and colleagues (2014) describe participatory peer-to-peer educational workshops in the UK that involved the training of local Somali women as community champions in Tower Hamlets, London [40]. These workshops covered a wide range of FGM related topics, including health and well-being, FGM legislation, and stories from women who had experienced FGM. A before and after workshop assessment to assess knowledge and attitudes was conducted that showed increased changes in knowledge acquisition, attitude, beliefs and intention relating to FGM. Additionally, one-on-one outreach sessions that involved 21 visits by either a project co-ordinator or the community champions to households to speak to families and to provide on-going support to the households allowed women to speak in confidence about FGM issues.

Two studies [41, 42] conducted in Canada and Australia report on the outcomes of broad public education campaigns that focused on improving community members and health professionals awareness of human rights issues and knowledge of FGM prevention. Daniel and colleagues (2011) describe a 10 weeks program of educational, health and sociocultural support sessions to discuss, compare and share stories targeting newly arrived African migrant and refugee women in Winnipeg, Canada. Moreover, a manual of materials for services in relation to sexual and reproductive health and FGM information and prevention was developed. They reported that many of the women felt that by virtue of living in a new culture, the decision not to circumcise daughters was easier [42]. Scott and Jerse (2011) reported that educational interventions in Australia may have some effect in changing attitudes and knowledge on FGM of migrant communities to abandon the practice, especially when community champions and bilingual community workers from the affected communities are actively engaged [41]. Similarly, Brown and colleagues in their UK studies showed that when the community is engaged in the design of outreach interventions for girls, women and men, awareness of FGM can be enhanced, enabling participants to talk freely about what had previously been considered a taboo topic [34, 35]. Community outreach from dedicated FGM clinical services in the UK were also found to be useful in raising awareness $[44,39]$.

The European Institute for Gender Equality (EIGE) report [48] notes that Sweden, the Netherlands and Italy have prioritised funding for prevention activities, but the majority of European Union Member States have not and that prevention activities lack baseline data and are poorly evaluated.

Four of the studies [37, 38, 44, 48] provide some insight into European laws to prevent FGM and associated activities to educate communities about the laws and support health and other professionals to implement them. The EIGE report states that in 2013 nine of the 27 EU Member States had specific criminal law provisions on FGM. However, no EU Member State had a specific provision on international protection and FGM in its national legislation [48]. According to Leye and Deblonde (2004), national legislation is a useful first step towards preventing FGM as it encourages institutions to take subsequent measures to prevent FGM but alone is not sufficient to stop FGM [38]. There is no evidence that the implementation of a specific law is more successful than general criminal laws to prevent FGM. Specific laws include child protection laws that maybe be applied in cases of FGM and special provisions with regards to professional secrecy and disclosure, which may be applied to cases of performed or planned FGM. General criminal laws including articles of the penal code may be applied to FGM-related cases.

Despite the development of laws, three studies included in the review note that it was very challenging to enforce these laws. France and Italy were cited as the only countries to have had prosecutions and even then, they involved long protracted processes [37]. One of the two main barriers for the implementation of legislation is the identification of cases, which is principally obstructed by the lack of knowledge of FGM among professionals. The second important barrier is the complexity of finding sufficient evidence to bring a case to court. The authors recommend that to be effective, different sectors and the relevant professionals need to be properly trained and involved in this process to ensure the harmonized implementation of criminal and child protection laws. The EIGE and Johansen et al. studies indicate that most EU countries have laws to prosecute adults involved in procuring FGM for girls outside of a 
country's borders, although its implementation is not uniform across nations. However, significant gaps have been identified in the application of the extraterritoriality principle to protect HIC residents from being subjected to FGM overseas [33, 48].

The report by Leye and colleagues [37] includes an evaluation of a series information and training workshops for professionals from various sectors in five EU member countries to improve knowledge of the law as it relates to FGM. The evaluation suggests that building the capacities of various professionals involved in prevention, child protection, law enforcement and health such as social services, immigration officers, policemen, prosecutors, health providers and other professionals may lead to an increase in the number of prosecutions. In the EIGE study, outreach programs targeting parents, professionals and decision makers among FGM-practising communities were described. These activities involved education workshops and public forums that provided information about the penalties for perpetrators, the role of professionals in reporting of FGM and the role of extra-territoriality principle in the European Union, where courts can adjudicate on cases outside the territory of their country. Additionally, training of practitioners was delivered as part of the rollout of new guidelines on child protection laws. The study found that practitioners, and parents are becoming increasingly aware of the risk of prosecution for FGM [48]. The three studies discussing laws in this review suggest that legislation may work more effectively when viewed as a facilitator of protection against harmful practices and when used to conduct negotiations with the communities, health care workers and prosecutors.

\section{Interventions to support secondary prevention interventions}

Five documents report on efforts to support safeguarding by improving citizen reporting of girls they suspect may be at risk of FGM to authorities. For example, Brown and Porter [34] in their report describe collaborative efforts in the UK that brought together, people from the voluntary and community sector, statutory agencies such as Safeguarding Boards, Metropolitan Police and health professionals, to strengthen community-based preventive work. These efforts comprise information sharing and the development of reporting or knowledge or suspicion of FGM risk to the authorities [34]. The NHS study reports on an audit undertaken of Clinical Commissioning Groups (CCG), one of which is the Croydon CCG services [43]. In the studies reviewed, the main focus of the interventions was on safeguarding that included FGM, building partnerships and the capacity of professionals involved (social welfare authorities, child protection officers, police officers, immigration services, school managers, legal professionals' healthcare professionals), to improve protective mechanisms and engagement with communities and relevant social actors.

Training workshops and updates for professionals to strengthen mandatory reporting obligations to protect girls from FGM were described as an important adjunct to legislative actions. However, training for health, education and social workers and police across nations in the European Union was reported as haphazard and not conducted on a continuous, structured and nationwide basis. This is said to be hampered by the fact that in many Member States, health professionals cannot break their code of silence when the crime of FGM has already been performed, because FGM is not generally considered as a type of repetitive, recurrent child abuse [48].

McCracken and colleagues (2017) describe the development of pilot of FGM-specific assessment and intervention tools and protocols alongside training for relevant professionals. This was supported by community outreach activities to raise awareness of FGM-related issues, promote understanding of services and legislation. Health professionals provided talks at schools for pupils, parents, teachers and governors [39]. Other community-based efforts to facilitate these safeguarding measures include training FGM champions and creating safe spaces for girls and women at risk of FGM to talk about FGM. Bi-lingual community co-facilitators were engaged to communicate information about safeguarding in culturally appropriate ways and motivate community members to support the protection legislation and protocols [35].

\section{Interventions to support the tertiary prevention of FGM associated conditions}

Only one report [39] included an evaluation of education provided to pregnant women and their partners who are eligible for de-infibulation to prevent obstetric complications. McCracken and colleagues (2017) describe the FGM Early Intervention Model delivered across three local authority areas in London. The authors found that dedicated FGM clinics within hospital midwifery services played a pivotal role in identifying women with FGM so that women could be provided with appropriate counselling and information about the law and available supportive services. During the pilot phase of the program, 235 women were seen in the FGM clinics. Practitioners were trained how to conduct risk assessment, identify individual women's understandings of the practice and their education and information needs related to FGM and to report instances where children were believed to be at risk of FGM. Only immediate outcomes were reported, i.e. changes in health care professional knowledge and attitude, including their recognition of the need for sensitivity in direct work to 
avoid re-traumatising or alienating women who have undergone FGM, and other members of potentially-affected communities [39]. No information about changes in the knowledge and attitudes of women as a result of information provided by health care professionals is available.

\section{Mapping interventions according to mode and focus}

Mapping the interventions described in the papers included in this review according to mode and foci of Beattie's model [31], (see Fig. 2) provides insight into approaches taken to address FGM in HIC. The majority of the interventions (described in nine papers) occupy the top half of this framework and are authoritative in mode. In the top right quadrant, most focus on population level legal action, policy and reporting protocols for safeguarding girls to prevent FGM and prosecute those for procuring or intending to procure FGM. These include both primary and secondary prevention activities. Interventions described in six papers (in the top left quadrant) employ the authority of public-health expertise to re-direct the behaviour of community members and professionals in top-down prescriptive ways, such as the use of media to raise awareness, health education workshops and continuing professional development that are largely primary prevention strategies.

There are fewer interventions described in the reviewed papers that can be mapped to the negotiated lower half of the framework. Only one paper [39] describes individual personal counselling activities also noted

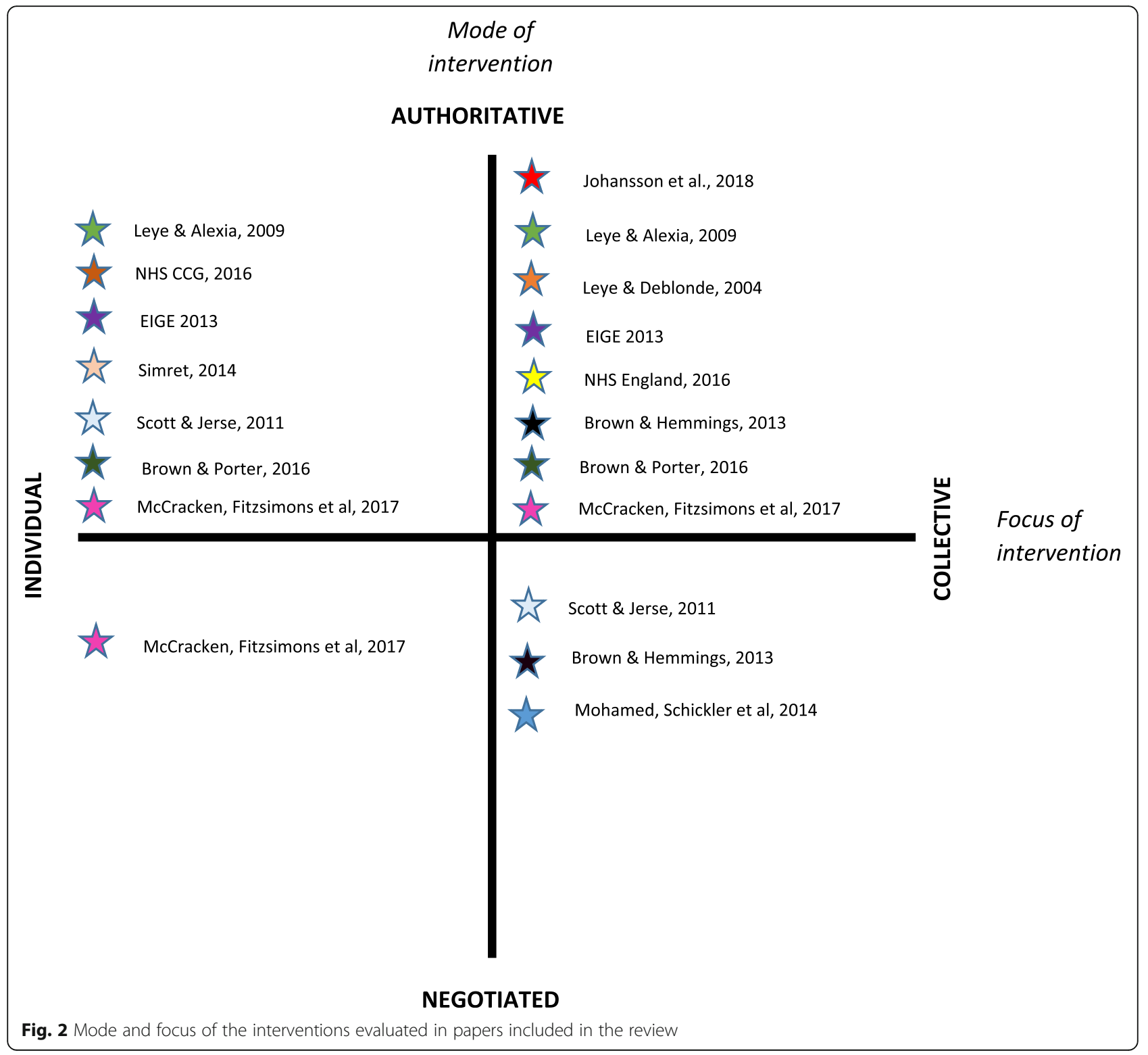


previously as tertiary prevention directed at women who have FGM. Three papers outline interventions that focus on collective community development activities that are focused on primary prevention [35, 40, 41].

Several papers describe interventions with multiple modes and foci and are therefore represented in more than one quadrant. Three documents in the review [34, 37, 48] identified activities that are authoritative in mode with activities that focus on individual or groups and populations. Two documents [35, 41] describe authoritative, individually focused education workshops, as well as collective community development strategies with affected communities involving community workers. McCracken et al. [39] includes activities across all but one paradigm. Scott and Jerse [41] describe both individual and collective education interventions to address FGM in two quadrants while McCracken et al., describe personal counselling, professional training and safeguarding protocols that occupy three quadrants.

\section{Discussion}

This review sought to gain insights into the characteristics of health interventions and associated outcomes. We examined programmatic approaches and strategies, target audiences and evaluation findings of interventions in HIC to improve the knowledge base to inform the design and evaluation of FGM health interventions. The majority of the reports included in this review described primary prevention activities in the UK and across Europe that are largely prescriptive or top-down forms of social intervention as compared with participative or 'bottom-up' forms. Emphasis is given to legislative action and strengthening the capacity of health care professionals, education and awareness raising for community members. This paradigm suggests the predominance of reformist and conservative ideologies and prescriptive structures. Beattie [31] depicts reformist legislative activities as "deprivation models" where bureaucratic interventions can address social injustice and work to protect those at risk of FGM. On the other hand, activities that favour health persuasion are focused on a "deficit model", where selected individuals whose FGM knowledge and practices are regarded as lacking, are corrected. These approaches contrast with the few bottom-up and participative approaches identified in this review. Three papers $[35,40,41]$ outline community development activities that support an "emancipation model" that mobilises community groups to design and deliver their own health promotion. One report identified tailored activities aimed at empowering individual women [39].

\section{Multifaceted comprehensive health promotion for FGM} While legislative action and health persuasion are important, the focus on these action paradigms do not appear to be justified by a strategic approach that is informed by baseline research. A focus on these paradigms may result in limited change and an unnecessary focus on risk factors that do not acknowledge the social determinants of health. Thomas and Stewart [49] have argued that a focus on these top-down approaches may lead to "victim blaming" and can work to disempower those most affected, in this case migrant and refugee women and girls from countries where FGM is practiced. FGM is a complex issue that requires a multifaceted response that encompasses socio-economic, physical and environmental factors, education and learning, services and facilities, and community activities. Beattie's model supports this concept and illustrates that approaches across all quadrants and axes are required for holistic, well-rounded health promotion policy and practice. A health promotion approach is therefore required that considers prevention activities as part of a holistic program of action to prevent FGM but also engage communities in positive health enhancement.

\section{Evidence to prevent FGM}

This review was not able to establish the effectiveness of one intervention over another, or the cumulative effects of one or several interventions on the prevalence of FGM. The evidence on the interventions outlined in the reports included in this review is therefore limited. The assessments are largely process evaluations that include measures of short-term outcomes or audits of practices. Much of the available evidence has focused on measuring immediate outputs such as pre and post intervention changes in attitude, knowledge and confidence. It points to the possibility of these interventions being useful in helping migrant communities abandon this harmful practice. Reports examining FGM legislation are largely descriptive overviews of the current legal and policy situation in HIC that has recently been captured in the World Bank's Compendium on international and national legal frameworks on FGM and in a recent text $[50,51]$. These evaluations have been hampered by a lack of monitoring and reporting systems [33] and the implementation of legislation, affected by a lack of clear guidelines, legal ambiguity, and reluctance to report parents and community members. A review by Balfour and others also shows that the evidence on educational/promotional interventions is weak and mainly focuses on surrogate markers of opinion change etc. [52]. This review included only peer reviewed evidence and as such included only two studies. This review has shown little insight into the long-term impact of single or multiple 
health prevention activities. Given the significant amount of effort and resources spent on programs specifically designed to promote the abandonment of FGM, there is need to strengthen research designs to include more robust methodologies, community driven, crossdisciplinary and international collaborative studies which take into consideration not just the immediate outcomes but the impact of the interventions.

\section{Involving women and girls in the design and evaluation of health prevention}

Our review located one report [39] that examined outcomes of an initiative that involved the training of health professionals to provide education and information during consultations with pregnant women who have experienced FGM. While this sheds some light on the importance of improving the skills of professionals to provide quality care as emphasised in WHO guidelines [1], evidence is needed concerning the experience of women and girls and their values and preferences during clinical consultations. A recent review has examined the maternity care experiences and health needs of women with FGM in HIC [53]. Another study on health information and education interventions for FGM included women's perspectives [54]. While the papers included in these reviews do not investigate the outcomes of specific interventions, rather current care, they do indicate that there is much to be done to better meet women's needs for health promotion. Both reviews highlight poor provider communication, negative attitudes and a lack of knowledge about FGM. If women centred, culturally appropriate health promotion including prevention is to be delivered during consultations that empowers women to take control over their own health, then women's needs must be considered in such interactions. Women should be involved in the design and evaluation of education and information resources [55].

\section{Multi-agency response to FGM}

The majority of the interventions in our review involve a wide range of professionals from multiple agencies, including health professionals, police, social workers and school teachers across the public and non-state sector. This cross-sector approach to FGM prevention was highlighted in the evaluations of safeguarding practices from the UK where collaboration was fostered through networking, linking national child protection policy with organization policy, and training initiatives. However, central to many of these efforts were activities to foster engagement with the local community through outreach and the involvement of community champions and advocates to raise awareness of safeguarding laws and reporting processes.
There are a number of documents to guideline multiagency response in HIC. In the UK, the Government has produced Multi-agency Statutory Guidelines for frontline professionals [56] working to safeguard children at risk from FGM in England and Wales. In Spain, an Action protocol for the prevention of FGM [57] lays out ways in which health care, education, social services, child care services and security professionals can collaborate to identify and report children at risk in Catalunya. Health professional themselves have also produced guidelines for inter-professional collaboration for safeguarding such as the Intercollegiate recommendations for identifying, recording and reporting in the UK [58]. In Australia, service coordination emphasises guidelines have been produced to facilitated collaborative partnerships between services and organisations in the community [59].

\section{Best practice health prevention guidelines for FGM}

The interventions in this review were largely guided by the law as it relates to FGM and clinical practice guidelines. However, no mention is made of guidelines in this area. In Australia, the National Education Toolkit for FGM/C Awareness (NETFA) Best Practice Guide provides nationally accepted benchmarks for culturally appropriate FGM prevention programs for community organisations [60]. These ten standards include the need for resources to be inclusive and interactive, relevant, accurate, respectful of human rights and cultural dignity and empowering. The European commission through the REPLACE Approach provides a toolkit or 'how to' guide for community members affected by FGM, and community leaders and organisations working with them to bring about an end to FGM in the EU. [18]. The REPLACE approach has three pillars that emphasise behavioural change that recognises the socio-cultural context and social norm transformation, the importance of engaging and working with communities and the need for evaluation to inform all stages of programming.

\section{Limitations}

One of the limitations of our study is paucity of peer reviewed evidence for the research topic. The majority of the evidence retrieved for this study is therefore from grey literature, specifically from programmatic evaluations undertaken by consultants. As such, quality assessment may be variable, affecting the analysis. However, two authors appraised the studies independently to ensure the quality of reporting, methodological, rigour and conceptual depth and breadth of all included studies. Secondly, given the heterogeneous nature of the retrieved evidence (methods, purposes, between programs, target audiences, duration and content), it was difficult to make firm conclusions, particularly as many of the 
outcomes and measures for determining effectiveness varied widely. However, the inclusion of individual case studies of programs, provides rich, descriptive evidence of interventions, a useful contribution to address the questions on how governments, public health entities and providers have attempted to address the FGM in HIC.

\section{Conclusion}

Studies show that current evaluations conducted in HIC are mainly of primary preventions and top-down approaches that seek to employ the authority of public health expertise to re-direct the behaviour of community members and professionals. Few interventions focused on collective activities within communities or were aimed at empowering individual women. To streamline all the efforts in practice, there is a clear need for an integrated health promotion approach to best organise the multifaceted, multilayered and often overlapping public health interventions to address the medical, social and cultural issues involved in curtailing FGM. Moreover, evidence of the effectiveness of interventions on reducing the prevalence of FGM is lacking highlighting the need for investment in impact evaluation and rigorous study designs.

Beattie's framework may provide a useful way of guiding and articulating negotiated and authoritative health promotion actions required to address FGM at national and local levels. Such a framework would promote partnerships, synergies and communication between all stakeholders including education, health, police, social care agencies and the community to improve awareness and support for women and girls. Co-ordination would also promote greater efficiency in the implementation of interventions and avoid duplication of efforts. In addition, a comprehensive approach could potentially improve protocols, guidelines and learning materials, foster consistent and rigorous data collection and reporting on FGM, including information about girls at risk of, or girls who have already undergone FGM. This information would equip frontline service and healthcare professionals to effectively protect and care for girls and women in regard to FGM, intervene and effectively meet mandatory reporting requirements. Such a framework would bring logic, clarity, comprehension, evidence and an economically more effective response for current and future health promotion interventions addressing FGM in $\mathrm{HIC}$

\section{Abbreviations}

AACODS: Accuracy, Coverage, Objectivity, Date, and Significance; CASP: Critical Appraisal Skills Programme; EBSCO: Elton B. Stephens Co.; FGM/ FGC: Female Genital Mutilation /Cutting; HIC: High Income Country; PubMED: MEDLINE database of references and abstracts on life sciences and biomedical

\section{Acknowledgements}

Not applicable

\section{Authors' contributions}

AD conceptualised the study. JK, CN and TE contributed to the screening, data extraction and analysis, AD and NV counterchecked the studies. CN, AD and JK drafted the paper. All authors commented on and approved the final manuscript.

\section{Funding}

The authors report no special funding.

Availability of data and materials

Data generated and analysed during the study are included in this manuscript and its supplementary information files.

Ethics approval and consent to participate

Ethical approval or informed consent is not applicable, as this is a systematic review.

Consent for publication

Not applicable

\section{Competing interests}

The authors declare that they have no competing interests.

\section{Author details}

${ }^{1}$ The Australian Centre for Public and Population Health Research, Faculty of Public Health, University of Technology Sydney, PO Box 123, Broadway, Sydney, NSW 2007, Australia. ${ }^{2}$ Ministry of Health, PO Box 30016-00100, Nairobi, Kenya. ${ }^{3}$ Community and Public Health, Technical University of Kenya, PO Box 52428-00202, Nairobi, Kenya. ${ }^{4}$ Sydney Medical School, The University of Sydney, 135 Macquarie St, Sydney, NSW 2000, Australia.

Received: 27 February 2019 Accepted: 9 July 2019

Published online: 22 July 2019

\section{References}

1. WHO. Guidelines on the management of health complications from female genital mutilation. Geneva: World Health Organization; 2016. http://apps. who.int/iris/bitstream/handle/10665/206437/9789241549646_eng.pdf; jsessionid $=23$ FBA5760C9BF5181C609DFB35BCD2A1? sequence $=1$

2. UNICEF. Female genital mutilation/cutting: a statistical overview and exploration of the dynamics of change, vol. 2013. New York: United Nations Children's Fund. p. 184-90. https://data.unicef.org/topic/child-protection/ female-genital-mutilation/\# edn1

3. Berg RC, Odgaard-Jensen J, Fretheim A, Underland V, Vist G. An updated systematic review and meta-analysis of the obstetric consequences of female genital mutilation/cutting. Obstet Gynecol Int. 2014;2014:542859.

4. Kimani SMJ, Njue C. Health Impacts of Female Genital Mutilation/Cutting: A synthesis of the evidence: Nairobi Population Council; 2016. https://www. popcouncil.org/uploads/pdfs/2016RH_HealthlmpactsFGMC.pdf

5. Reisel D, Creighton SM. Long term health consequences of female genital mutilation (FGM). Maturitas. 2015;80:48-51.

6. Kandala N-B, Ezejimofor MC, Uthman OA, Komba P. Secular trends in the prevalence of female genital mutilation/cutting among girls: a systematic analysis. BMJ Glob Health. 2018;3:e000549.

7. UNICEF. Female genital mutilation/cutting: what might the future hold? New York: UNICEF; 2014. https://www.unicef.org/media/files/FGM-C_Report_ 7_15_Final_LR.pdf

8. Koski A, Heymann J. Thirty-year trends in the prevalence and severity of female genital mutilation: a comparison of 22 countries. BMJ Glob Health. 2017;2:e000467

9. UNICEF. Female genital mutilation/cutting: a statistical overview and exploration of the dynamics of change. New York: UNICEF; 2013. https:// www.unicef.org/cbsc/files/UNICEF_FGM_report_July_2013_Hi_res.pdf

10. Taher M. Understanding Female Genital Cutting in the Dawoodi Bohra Community; an exploratory survey. Massachusetts: Sahiyo; 2017. https:// sahiyo.files.wordpress.com/2017/02/sahiyo_report_final-updatedbymt2.pdf. 
11. Berg RC, Denison E. A tradition in transition: factors perpetuating and hindering the continuance of female genital mutilation/cutting (FGM/C) summarized in a systematic review. Health Care Women Int. 2013;34:837-59.

12. Gele AA, Johansen EB, Sundby J. When female circumcision comes to the west: attitudes toward the practice among Somali immigrants in Oslo. BMC Public Health. 2012;12:697.

13. Gele AA, Kumar B, Hjelde KH, Sundby J. Attitudes toward female circumcision among Somali immigrants in Oslo: a qualitative study. Int J Women's Health. 2012:4:7-17.

14. Johnsdotter S, Moussa K, Carlbom A, Aregai R, Essen B. "Never my daughters": a qualitative study regarding attitude change toward female genital cutting among Ethiopian and Eritrean families in Sweden. Health Care Women Int. 2009:30:114-33.

15. Johnson-Agbakwu CE, Helm T, Killawi A, Padela Al. Perceptions of obstetrical interventions and female genital cutting: insights of men in a Somali refugee community. Ethn Health. 2014;19:440-57.

16. Mitike G, Deressa W. Prevalence and associated factors of female genital mutilation among Somali refugees in eastern Ethiopia: a cross-sectional study. BMC Public Health. 2009;9:264.

17. Varol N, Hall JJ, Black K, Turkmani S, Dawson A. Evidence-based policy responses to strengthen health, community and legislative systems that care for women in Australia with female genital mutilation / cutting. Reprod Health. 2017:14:63.

18. Barrett HR, Brown K, Alhassan Y, Beecham D. The REPLACE approach: supporting communities to end FGM in the EU. Community handbook. Brussels: European Union; 2015. http://www.replacefgm2.eu/toolkit/default. aspx?section $=50$

19. Goldberg H, Stupp P, Okoroh E, Besera G, Goodman D, Danel I. Female genital mutilation/cutting in the United States: updated estimates of women and girls at risk, 2012. Public Health Rep. 2016;131:340-7.

20. Macfarlane A, Dorkenoo E. Prevalence of female genital mutilation in England and Wales: national and local estimates. London: City University of London; 2015. https://www.city.ac.uk/_data/assets/pdf_file/0004/282388/ FGM-statistics-final-report-21-07-15-released-text.pdf

21. Dawson A, Homer CSE, Turkmani S, Black K, Varol N. A systematic review of doctors' experiences and needs to support the care of women with female genital mutilation. Int J Gynaecol Obstet. 2015;131:35-40.

22. Dawson A, Turkmani S, Fray S, Nanayakkara S, Varol N, Homer C. Evidence to inform education, training and supportive work environments for midwives involved in the care of women with female genital mutilation: a review of global experience. Midwifery. 2015;31:229-38.

23. UK Department of Health. FGM Safeguarding and Risk Assessment Quick guide for health professionals. London: Government of the United Kingdom; 2017. https://assets.publishing.service.gov.uk/government/ uploads/system/uploads/attachment_data/file/585083/FGM_safeguarding_ and_risk_assessment.pdf. (Health Do ed

24. Berg RC, Denison E. Effectiveness of interventions designed to prevent female genital mutilation/cutting: a systematic review. Stud Fam Plan. 2012; 43:135-46.

25. Berg RC, Denison EM. A realist synthesis of controlled studies to determine the effectiveness of interventions to prevent genital cutting of girls. Paediatr Int Child Health. 2013;33:322-33.

26. Johansen REB, Diop NJ, Laverack G, Leye E. What works and what does not: a discussion of popular approaches for the abandonment of female genital mutilation. Obstet Gynecol Int. 2013;2013:348248.

27. Waigwa S, Doos L, Bradbury-Jones C, Taylor J. Effectiveness of health education as an intervention designed to prevent female genital mutilation/cutting (FGM/C): a systematic review. Reprod Health. 2018;15:62.

28. World Bank Country and Lending Groups: Country Classification 2018. https://datahelpdesk.worldbank.org/knowledgebase/articles/906519-worldbank-country-and-lending-groups

29. Tannahill A. Health promotion: the Tannahill model revisited. Public Health. 2008;122:1387-91.

30. Tengland P-AJHCA. Health promotion and disease prevention: logically different conceptions? vol. 18; 2010. p. 323-41.

31. Beattie A. Knowledge and control in health promotion: a test case for social policy and social theory. In: Gabe J, Calnan M, Bury M, editors. The sociology of the health service. London: Routledge; 1991. p. 162-202.

32. Moher D, Liberati A, Tetzlaff J, Altman D, Group. TP: preferred reporting items for systematic reviews and meta-analyses: the PRISMA statement. PLoS Med. 2009;6:e1000097.
33. Johansen REB, Ziyada MM, Shell-Duncan B, Kaplan AM, Leye E. Health sector involvement in the management of female genital mutilation/cutting in 30 countries. BMC Health Serv Res. 2018;18:240.

34. Brown E, Porter C. Evaluation of FGM prevention among communities affected by FGM: a participatory ethnographic evaluation research (PEER) study. London: Trust for London, Rosa, Comic Relief, Esmee Fairbairn, Options; 2016. http://www.rosauk.org/wp-content/uploads/2016/07/PEERFull-Report-.pdf

35. Brown E, Hemmings J. The FGM initiative: evaluation of the first phase (2010-2013). London: Trust for London, Rosa, Comic Relief, Esmee Fairbairn, Options; 2013. http://cdn.basw.co.uk/upload/basw_11339-9.pdf

36. EIGE - European Institute for Gender Equality. Female genital mutilation in the European Union and Croatia Vilnius: ElGE; 2013.

37. Leye E, Alexia S. Responding to female genital mutilation striking the right balance in Europe between prosecution and prevention : a review of legislation, vol. 80. Ghent: EC Daphne Programme, ICRH Ghent University; 2009. p. 80.

38. Leye $\mathrm{E}$, Deblonde J. A comparative analysis of the different legal approaches towards female genital mutilation in the $15 \mathrm{EU}$ member states, and the respective judicial outcomes in Belgium, France, Spain, Sweden and the UK. Ghent: International Centre for Reproductive Health; 2004. https:// www.researchgate.net/publication/228775749_A_comparative_analysis_of_ the_different_legal_approaches_towards_female_genital_mutilation_in_ the_15_EU_Member_States_and_the_respective_judicial_outcomes_in_ Belgium_France_Spain_Sweden_and_the_UK

39. McCracken K, FitzSimons A, Priest S, Torchia K. The Mayor's Office for Policing and Crime Female Genital Mutilation Early Intervention Model: an evaluation. London: Department of Education, Government of the United Kingdom; 2017. https://assets.publishing.service.gov.uk/government/ uploads/system/uploads/attachment_data/file/585172/Female_genital_ mutilation_early_intervention_model_evaluation.pdf

40. Mohamed N, Schickler P, Warsame Z, Glew C. A Report on Participatory Workshops on FGM/C (Female Genital Mutilation/Cutting) with the Somali Community in Tower Hamlets. London: Women's Health \& Family Services; 2014. http://whfs.org.uk/index.php/what-we-do/fgm/fgm-c-peer-support

41. Scott M, Sd J. Female Genital Mutilation Education Program Evaluation Final report. Sydney: WestWood Spice; 2011. http://www.wslhd.health.nsw.gov. au/ArticleDocuments/2228/TAB\%20A\%20\%20final\%20report\%20on\%2 0the\%20Evaluation\%20ofthe\%20FGM\%20Education\%20Program.pdf.aspx

42. Simret D. Our selves, our daughters:community-based education and engagement addressing female genital cutting (FGC) with refugee and immigrant African women in Winnipeg 2012-13. Winnipeg: Sexuality Education Resource Centre; 2014. https://serc.mb.ca/wp-content/uploads/2 018/08/Our-Selves-Our-Daughters-2012-13-Final-Report.pdf

43. NHS England. NHS England London Region Clinical Commissioning Group (CCG) Safeguarding Deep Dive 2015/16 Report. London: NHS England; 2016 https://londonadass.org.uk/wp-content/uploads/2014/12/Final-NHS-EnglandLondon-CCG-Safeguarding-Deep-Dive-Report-2016.pdf

44. NHS Croydon Clinical Commissioning Group. Project: female genital mutilation (FGM) July 2016. Croydon: NHS Croydon Clinical commissioning group; 2016.

45. AACODS checklist for appraising grey literature https://dspace.flinders.edu. au/xmlui/bitstream/handle/2328/3326/AACODS_Checklist.pdf;jsessionid= AB42D4A4AEAC2295306ACA74417510AA?sequence $=4$. https://dspace. flinders.edu.au/jspui/bitstream/2328/3326/4/AACODS_Checklist.pdf, Accessed 29 Mar 2017).

46. Critical Assessment Skills Programme: CASP (Systematic Review, Qualitative, Cohort Study, Case Control) Checklist. 2018.

47. Schneider MJ, Schneider H. Introduction to public health. Burlington: Jones \& Bartlett Publishers; 2017

48. European Institute for Gender Equality. Female genital mutilation in the European Union and Croatia. Brussels: Equality, European Institute for Gender; 2013.

49. Thomas S, Stewart J. Optimising health promotion activities. J Community Nurs. 2005:19:9-12.

50. World Bank. Compendium on international and National Legal Frameworks on female genital mutilation. 2nd ed. Washington, DC: World Bank; 2018. http://documents.worldbank.org/curated/en/828661517490252879/ Compendium-of-international-and-national-legal-frameworks-on-femalegenital-mutilation

51. Kandala N-B, Komba PN. Female Genital Mutilation Around The World Switzerland: Springer; 2018. 
52. Balfour J, Abdulcadir J, Say L, Hindin MJ. Interventions for healthcare providers to improve treatment and prevention of female genital mutilation: a systematic review. BMC Health Serv Res. 2016;16:409.

53. Turkmani S, Homer CSE, Dawson A. Maternity care experiences and health needs of migrant women from female genital mutilation-practicing countries in high-income contexts: a systematic review and meta-synthesis. Birth. 2018;0:3-14.

54. Smith $\mathrm{H}$, Stein $\mathrm{K}$. Health information interventions for female genital mutilation. Int J Gynecol Obstet. 2017;136:79-82.

55. Green JM. Integrating women's views into maternity care research and practice. Birth. Nov 2012;39:291-5.

56. UK Government. Multi-agency statutory guidance on female genital mutilation. London: Government of the United Kingdom; 2016. https:// www.gov.uk/government/publications/multi-agency-statutory-guidance-onfemale-genital-mutilation

57. Generalitat de Catalunya. Action protocol for the prevention of FGM. Barcelona: Generalitat de Catalunya, Department of Social Action and Civic Responsibility Secretariat of Immigration; 2007. http://treballiaferssocials. gencat.cat/web/.content/03ambits_tematics/05immigracio_refugi/ 08recursosprofessionals/02prevenciomutilaciofemenina/Protocol_mutilacio_ angles.pdf

58. RCM RCN RCOG Equality now UNITE. Tackling FGM in the UK intercollegiate recommendations for identifying, recording and reporting. https://www.rcn.org.uk/professional-development/publications/pub-004531. London: Royal College of Midwives; 2013.

59. Jordan L. Improving the health care of women and girls affected by female genital mutilation/ cutting: a national approach to service coordination. Box Hill: Family Planning Victoria; 2013. https://www.fpv.org.au/assets/resources/ FGM-ServeCoOrdinationGuideNationalWeb.pdf

60. MCWH. NETFA National Standards Framework for FGM/C-related educational resources. Melbourne: Multicultural Centre for Women's Health; 2014. http://www.netfa.com.au/downloads/NETFA\%202015\%20National\%2 0Standards\%20Framework.pdf.

\section{Publisher's Note}

Springer Nature remains neutral with regard to jurisdictional claims in published maps and institutional affiliations.

Ready to submit your research? Choose BMC and benefit from:

- fast, convenient online submission

- thorough peer review by experienced researchers in your field

- rapid publication on acceptance

- support for research data, including large and complex data types

- gold Open Access which fosters wider collaboration and increased citations

- maximum visibility for your research: over $100 \mathrm{M}$ website views per year

At $\mathrm{BMC}$, research is always in progress.

Learn more biomedcentral.com/submissions 